\title{
A LOCAL STRONG SOLUTION OF THE NAVIER-STOKES PROBLEM IN $L_{2}(\Omega)$
}

\section{MAOTING TONG ${ }^{1}$ and DAORONG TON ${ }^{2}$}

${ }^{1}$ Department of Mathematical Science

Xi'an Jiaotong-Liverpool University

Suzhou, 215123

P. R. China

e-mail: Maoting.Tong18@student.xjtlu.edu.cn

2Department of Mathematics

Hohai University

Nanjing, 210098

P. R. China

Current address:

1-3306 Moonlight Square

Nanjing, 210036

P. R. China

e-mail: 1760724097@qq.com

2010 Mathematics Subject Classification: Primary 35Q30, 76N10; Secondary 47D06.

Keywords and phrases: Navier-Stokes equation, existence and uniqueness, local solution, semigroup of operators, invariance, Banach lattice, fractional powers.

The first author is grateful to her supervisors Chi-Kun Lin and Xinyao Yang for their teaching and cultivation.

Received March 21, 2020; Revised May 8, 2020

(C) 2020 Scientific Advances Publishers 


\begin{abstract}
In this paper, we prove that the Navier-Stokes initial value problem (1) has a unique smooth local strong solution $u(t, x)$ and $p(t, x)$ if the following condition are satisfied:

(1) $f \in D L_{2}(\Omega)$ and $f$ is Hölder continuous about $t$ on $(0, \infty)$;

(2) The initial value $u_{0} \in D\left((-\Delta)^{\frac{1}{2}}\right)$ is smooth, divergence-free vector field.
\end{abstract}

The Navier-Stokes initial value problem can be written in its classical form as

$$
\left\{\begin{array}{c}
\frac{\partial u}{\partial t}=\Delta u-\nabla p-(u \bullet \nabla) u+f, x \in \Omega, t \in(0, \infty), \\
\nabla \bullet u \equiv \operatorname{div} u=0, \\
\left.u\right|_{\partial \Omega}=0, t \in(0, \infty), \\
\left.u\right|_{t=0}=u_{0}, x \in \Omega,
\end{array}\right.
$$

where $\Omega$ is a bounded domain in $R^{3}$ with smooth boundary $\partial \Omega$ of class $C^{3}, u=u(t, x)=\left(u_{1}(t, x), u_{2}(t, x), u_{3}(t, x)\right)$ is the velocity field, $u_{0}=u_{0}(x)$ is the initial velocity, $p=p(t, x)$ is the pressure, $f=f(t, x)$ is the external force,

$$
\Delta u=\left(\sum_{i=1}^{3} \frac{\partial^{2} u_{1}}{\partial x_{i}^{2}}, \sum_{i=1}^{3} \frac{\partial^{2} u_{2}}{\partial x_{i}^{2}}, \sum_{i=1}^{3} \frac{\partial^{2} u_{3}}{\partial x_{i}^{2}}\right) .
$$

In these four equations $u, p$ are unknown and $f, u_{0}$ are given. The boundary condition imposed on the velocity at $\Omega$ is homogeneous.

The existence, uniqueness, and regularity properties of solutions for the Navier-Stokes problems are extensively studied. There is an extensive literature on the solvability of the initial value problem for Navier-Stokes equations. 
Let $L_{2}(\Omega)$ be the Banach space of real vector functions in $L^{2}(\Omega)$. That is,

$$
L_{2}(\Omega)=\left\{h: \Omega \rightarrow R^{3}, h=\left(h_{1}, h_{2}, h_{3}\right), h_{i} \in L^{2}(\Omega)(i=1,2,3)\right\} .
$$

For $u=\left(u_{1}, u_{2}, u_{3}\right) \in L_{2}(\Omega)$, we define the norm

$$
\|u\|_{L_{2}(\Omega)}=\left(\sum_{i=1}^{3}\left\|u_{i}\right\|_{L^{2}(\Omega)}^{2}\right)^{\frac{1}{2}}
$$

then $L_{2}(\Omega)$ is a Banach space. The set of all real vector functions $u$ such that $\operatorname{div} u=0$ and $u \in C_{0}^{\infty}(\Omega)$ is denoted by $C_{0, \sigma}^{\infty}(\Omega)$. Let $D L_{2}(\Omega)$ be the closure of $C_{0, \sigma}^{\infty}(\Omega)$ in $L_{2}(\Omega)$. If $u \in C^{\infty}(\Omega)$, then

$u \in D L_{2}(\Omega)$ if and only if $\operatorname{div} u=0$ in $\Omega$ and $u_{n}=0$ on $\partial \Omega$ (p. 270 in [3]).

In this paper, we always consider the spaces of vector value functions on $\Omega$.

We have

$$
\begin{gathered}
D L_{2}(\Omega) \subset L_{2}(\Omega)=W^{0,2}(\Omega), \quad\|\bullet\|_{D L_{2}(\Omega)}=\|\bullet\|_{L_{2}(\Omega)}, \\
L_{2}(\Omega)=D L_{2}(\Omega) \oplus D L_{2}(\Omega)^{\perp} .
\end{gathered}
$$

From [2] and [3], we see that $D L_{2}(\Omega)^{\perp}=\left\{\nabla h ; h \in W^{1,2}(\Omega)\right\}$. Let $P$ be the orthogonal projection from $L_{2}(\Omega)$ onto $D L_{2}(\Omega) . A=-P \Delta$ is called the Stokes operator. By applying $P$ to the first equation of (1.1) and taking account of the other equations, we are let the following abstract initial value problem, Pr. II

$$
\left\{\begin{array}{c}
\frac{d u}{d t}=P \Delta u+F u+P f, t \in(0, \infty), \\
\left.u\right|_{t=0}=u_{0}, x \in \Omega,
\end{array}\right.
$$

where $F u=-P(u \bullet \nabla) u$. 
We consider Equation (3) in integral form Pr. III

$$
u(t)=e^{t P \Delta} u_{0}+\int_{0}^{t} e^{(t-s) P \Delta} F u(s) d s
$$

In Theorem 1.6 of [3], Fujita and Kato proved that if $u$ is a solution of (3) then $u$ is of the class $C^{\infty}$ as $L_{2}(\Omega)$-valued functions. They gave some sufficient conditions for (3) having a solution. In Theorem 3.4 of [2], Giga and Miyakawa proved that the solution of (4) belongs to $\left(C^{\infty}(\bar{\Omega} \times(0, T])\right)^{n}$. They gave some sufficient conditions for (3) and (4) having a solution. In [8], Otelbaev proved that if all $\frac{\partial u}{\partial t}, \Delta u$, $(u \bullet \nabla) u$, div $p \in L_{2}((0, T) \times \Omega)$, then (1) have the unique local solution. But he did not prove that this solution is smooth. In [10], Shakhmurov discussed nonlocal Navier-Stokes problems in abstract function space $D L_{2}(\Omega)$. He gave some sufficient conditions for (4) having a solution. In this paper, we will prove that the Navier-Stokes initial value problem (1) has the unique local strong solution $u(t, x) \in\left(C^{\infty}\left(\left[0, t_{1}\right] \times \Omega\right)\right)^{3}$, $p(t, x) \in C^{\infty}\left(\left[0, t_{1}\right] \times \Omega\right)$ in the state space $D L_{2}(\Omega)$ by using the theory of semigroup of operators.

For $u=\left(u_{1}, u_{2}, u_{3}\right) \in L_{2}(\Omega)$ we define $\Delta u=\left(\Delta u_{1}, \Delta u_{2}, \Delta u_{3}\right)$ and $\nabla u=\left(\frac{\partial u_{1}}{\partial x_{1}}, \frac{\partial u_{2}}{\partial x_{2}}, \frac{\partial u_{3}}{\partial x_{3}}\right)$. Since the operator $-\nabla=-\sum_{i=1}^{3} \frac{\partial^{2}}{\partial x_{i}^{2}}$ is strongly elliptic of order 2. Theorem 7.3.6 in [11] implies that $\Delta$ is the infinitesimal generator of an analytic semigroup of contractions on $L^{2}(\Omega)$ with $D(\Delta)=H^{2}(\Omega) \cap H_{0}^{1}(\Omega)$. Hence $\Delta$ is also the infinitesimal generator of an analytic semigroup of contraction on $L_{2}(\Omega)$ with $D(\Delta)=H_{2}(\Omega) \cap H_{1,0}(\Omega)$, where $H_{2}(\Omega)$ and $H_{1,0}(\Omega)$ are the Sobolev spaces of vector value in $H^{2}(\Omega)$ and $H_{0}^{1}(\Omega)$, respectively. We will prove that $\Delta$ is also the infinitesimal generator of an analytic semigroup of contraction on $D L_{2}(\Omega)$. 
An operator $A$ is called preserving divergence-free on a vector value functions space $X$ if $A$ maps every $u \in X$ with $\operatorname{div} u=0$ to an $A u$ with $\operatorname{div} A u=0$.

Lemma 1. For every $u \in L_{2}(\Omega), \operatorname{div} u=0$ if and only if $\operatorname{div}(\lambda I-\Delta)$ $u=0$ for

$$
\lambda \in \sum_{\vartheta}=\{\lambda: \vartheta-\pi<\arg \lambda<\pi-\vartheta,|\lambda| \geq r\},
$$

where $0<\vartheta<\pi / 2$.

Proof. Let $u=\left(u_{1}, u_{2}, u_{3}\right) \in L_{2}(\Omega)$. Then

$$
\begin{aligned}
\Delta u=( & \left.\frac{\partial^{2} u_{1}}{\partial x_{1}{ }^{2}}+\frac{\partial^{2} u_{1}}{\partial x_{2}{ }^{2}}+\frac{\partial^{2} u_{1}}{\partial x_{3}{ }^{2}}, \frac{\partial^{2} u_{2}}{\partial x_{1}{ }^{2}}+\frac{\partial^{2} u_{2}}{\partial x_{2}{ }^{2}}+\frac{\partial^{2} u_{2}}{\partial x_{3}{ }^{2}}, \frac{\partial^{2} u_{3}}{\partial x_{1}{ }^{2}}+\frac{\partial^{2} u_{3}}{\partial x_{2}{ }^{2}}+\frac{\partial^{2} u_{3}}{\partial x_{3}{ }^{2}}\right) \\
\operatorname{div}(\Delta u)= & \frac{\partial^{3} u_{1}}{\partial x_{1}{ }^{3}}+\frac{\partial^{3} u_{1}}{\partial x_{1} \partial x_{2}{ }^{2}}+\frac{\partial^{3} u_{1}}{\partial x_{1} \partial x_{3}{ }^{2}}+\frac{\partial^{3} u_{2}}{\partial x_{2} \partial x_{1}{ }^{2}}+\frac{\partial^{3} u_{2}}{\partial x_{2}{ }^{3}} \\
& +\frac{\partial^{3} u_{2}}{\partial x_{2} \partial x_{3}{ }^{2}}+\frac{\partial^{3} u_{3}}{\partial x_{3} \partial x_{1}{ }^{2}}+\frac{\partial^{3} u_{3}}{\partial x_{3} \partial x_{2}{ }^{2}}+\frac{\partial^{3} u_{3}}{\partial x_{3}{ }^{3}} \\
= & \frac{\partial^{2}}{\partial x_{1}{ }^{2}}\left(\frac{\partial u_{1}}{\partial x_{1}}+\frac{\partial u_{2}}{\partial x_{2}}+\frac{\partial u_{3}}{\partial x_{3}}\right)+\frac{\partial^{2}}{\partial x_{2}{ }^{2}}\left(\frac{\partial u_{1}}{\partial x_{1}}+\frac{\partial u_{2}}{\partial x_{2}}+\frac{\partial u_{3}}{\partial x_{3}}\right) \\
& +\frac{\partial^{2}}{\partial x_{3}^{2}}\left(\frac{\partial u_{1}}{\partial x_{1}}+\frac{\partial u_{2}}{\partial x_{2}}+\frac{\partial u_{3}}{\partial x_{3}}\right) \\
= & \left(\frac{\partial^{2}}{\partial x_{1}{ }^{2}}+\frac{\partial^{2}}{\partial x_{2}{ }^{2}}+\frac{\partial^{2}}{\partial x_{3}{ }^{2}}\right)\left(\frac{\partial u_{1}}{\partial x_{1}}+\frac{\partial u_{2}}{\partial x_{2}}+\frac{\partial u_{3}}{\partial x_{3}}\right)=\Delta(\operatorname{div} u) .
\end{aligned}
$$

So we have

$$
\operatorname{div}[(\lambda I-\Delta) u]=(\lambda I-\Delta)(\operatorname{div} u)
$$

From (5), it is clear that $\operatorname{div} u=0$ implies that $\operatorname{div}(\lambda I-\Delta) u=0$. 
Since $-\Delta$ is a strongly elliptic operator of order 2 on $\Omega$. From Theorem 7.3.2 in [11], it follows that there exist constant $C>0, R \geq 0$ and $0<\vartheta<\pi / 2$ such that

$$
\|u\|_{L_{2}(\Omega)} \leq \frac{C}{|\lambda|}\|(\lambda I-\Delta) u\|_{L_{2}(\Omega)}
$$

for $u \in D(\Delta)=H_{2}(\Omega) \cap H_{1,0}(\Omega) \subset L_{2}(\Omega)$ and

$$
\lambda \in \sum_{\vartheta}=\{\lambda: \vartheta-\pi<\arg \lambda<\pi-\vartheta,|\lambda| \geq r\} .
$$

From (6), it follows that for every $\lambda \in \sum_{\vartheta}$ the operator $\lambda I-\Delta$ is injective from $D(\Delta)$ into $L_{2}(\Omega)$. From (5), it follows that $\operatorname{div}(\lambda I-\Delta) u=0$ implies that $\operatorname{div} u=0$.

Lemma 2 (1.5.12 in [5]). Let $\{T(t): t \geq 0\}$ be a $C_{0}$-semigroup on a Banach space $X$. If $Y$ is a closed subspace of $X$ such that $T(t) Y \subset Y$ for all $t \geq 0$, i.e., if $Y$ is $T(t)_{t \geq 0}$-invariant, then the restrictions

$$
T(t)_{\mid}:=T(t)_{\mid Y}
$$

form a $C_{0}$-semigroup $\left\{T(t)_{\mid}: t \geq 0\right\}$, called the subspace semigroup, on the Banach space $Y$.

Lemma 3 (Proposition 2.2.3 in [5]). Let $(A, D(A))$ be the generator of a $C_{0}$-semigroup $\{T(t): t \geq 0\}$ on a Banach space $X$ and assume that the restricted semigroup (subspace semigroup) $\left\{T(t)_{\mid}: t \geq 0\right\}$ is a $C_{0}$-semigroup on some $(T(t))_{t \geq 0}$-invariant Banach space $Y \rightarrow X$. Then the generator of $\left\{T(t)_{\mid}: t \geq 0\right\}$ is the part $\left(A_{\mid}, D\left(A_{\mid}\right)\right)$of $A$ in $Y$.

Lemma 4. The operator $\Delta$ with $D(\Delta) \subset D L_{2}(\Omega)$ is the infinitesimal generator of an analytic semigroup of contractions on $D L_{2}(\Omega)$. 
Proof. From Theorem 7.3.6 in [11] $\Delta$ is the infinitesimal generator of an analytic semigroup of contractions on $L^{2}(\Omega)$. Then $\Delta$ is also the infinitesimal generator of an analytic semigoup of contractions on $L_{2}(\Omega)$. Let $\{T(t) \mid t \geq 0\}$ be the restriction of the analytic semigroup generated by $\Delta$ on $L_{2}(\Omega)$ to the real axis, $\{T(t) \mid t \geq 0\}$ is a $C_{0}$ semigroup of contractions by Theorem 7.2.5 and Theorem 3.1.1 in [11]. We have already noted that $D L_{2}(\Omega)$ is a closed subspace of $L_{2}(\Omega)$ and is also a Hilbert space. We want to show that $D L_{2}(\Omega)$ is $T(t)_{t \geq 0}$-invariant. For every $u \in L_{2}(\Omega)$ with $\operatorname{div} u=0$ and $\lambda \in \rho(\Delta) \cap \sum_{\vartheta}=\{\lambda: \vartheta-\pi<\arg$ $\lambda<\pi-\vartheta,|\lambda| \geq r\}$ we have $(\lambda I-\Delta)[R(\lambda: \Delta) u]=u$, where $\sum_{\vartheta}$ is the same as in the proof of Lemma 1. From Lemma 1 it follows that $\operatorname{div} R$ $(\lambda: \Delta) u=0$. That is to say that $R(\lambda: \Delta)$ is preserving divergence-free for $\lambda \in \rho(\Delta) \cap \sum_{\vartheta}$. From Theorem 2.5.2(c) in [11] it follows that $\rho(\Delta) \supset R^{+}$, and so $\rho(\Delta) \cap \sum_{\vartheta} \supset\{\lambda: \lambda \geq r\}$. Hence $R(\lambda: \Delta)$ is preserving divergence free for every $\lambda \geq r$. Let $u \in D L_{2}(\Omega)$ then there exists a sequence $u_{n}$ such that $\lim _{n \rightarrow \infty} u_{n}=u$ and $\operatorname{div} u_{n}=0$ for $n=1,2, \ldots$. Since $R(\lambda: \Delta)$ is bounded and so is continuous. Hence $\lim _{n \rightarrow \infty} R(\lambda: \Delta) u_{n}=R(\lambda: \Delta) u$ and $\operatorname{div} R(\lambda: \Delta) u_{n}=0$ for every $\lambda \geq r$. Therefore $R(\lambda: \Delta) u \in D L_{2}(\Omega)$ for every $\lambda \geq r$. It follows that $D L_{2}(\Omega)$ is $R(\lambda: \Delta)$-invariant for every $\lambda \geq r$. Now, the Theorem 4.5.1 in [11] implies that $D L_{2}(\Omega)$ is $T(t)_{t \geq 0}$-invariant. From Lemmas 2 and 3 , it follows that $\Delta_{\mid D L_{2}(\Omega)}$ with $D\left(\Delta_{\mid D L_{2}(\Omega)}\right)=D(\Delta) \cap D L_{2}(\Omega)$ is the infinitesimal generator of the $C_{0}$ semigroup $\left\{T(t)_{\mid D L_{2}(\Omega)}: t \geq 0\right\}$ of contractions on $D L_{2}(\Omega)$. 
We will prove that $\left\{T(t)_{\mid D L_{2}(\Omega)}: t \geq 0\right\}$ can also be extended to an analytic semigroup on $D L_{2}(\Omega)$. Suppose that $\lambda \in \rho(\Delta) \cap \sum_{\vartheta}$, i.e., there exists $R(\lambda: \Delta)$ from $L_{2}(\Omega)$ into $D(\Delta)$. Then for any $u \in D L_{2}(\Omega) \subset L_{2}(\Omega)$, $R(\lambda: \Delta) u \in D L_{2}(\Omega)$. We have

$$
(\lambda I-\Delta) R(\lambda: \Delta) u=u \quad \text { and } \quad R(\lambda: \Delta)(\lambda I-\Delta) u=u .
$$

Since $R(\lambda: \Delta)$ is preserving divergence-free we have

$$
R(\lambda: \Delta) u \in D L_{2}(\Omega) \quad \text { and } \quad(\lambda I-\Delta) u \in D L_{2}(\Omega) .
$$

Thus the formula (7) becomes

$$
\left(\lambda I-\Delta_{\mid D L_{2}(\Omega)}\right) R(\lambda: \Delta) u=u \quad \text { and } \quad R(\lambda: \Delta)\left(\lambda I-\Delta_{\mid D L_{2}(\Omega)}\right) u=u .
$$

Hence $\left(\lambda I-\Delta_{\mid D L_{2}(\Omega)}\right) R(\lambda: \Delta)=I$ and $R(\lambda: \Delta)\left(\lambda I-\Delta_{\mid D L_{2}(\Omega)}\right)=I$. We get

$$
\left(\lambda I-\Delta_{\mid D L_{2}(\Omega)}\right)^{-1}=R(\lambda: \Delta)_{\mid D L_{2}(\Omega)}
$$

From the formula (8) and Theorem 2.5.2(c) in [11], we have

$$
\begin{gathered}
\rho\left(\Delta_{\mid D L_{2}(\Omega)}\right) \supset \rho(\Delta) \cap \sum_{\vartheta} \supset \sum=\left(\{\lambda:|\arg \lambda|\langle\pi / 2+\delta\} \cup\{0\}) \cap \sum_{\vartheta}\right. \\
=\sum_{\vartheta}=\left\{\lambda:|\arg \lambda|_{<} \vartheta_{1},|\lambda| \geq r\right\},
\end{gathered}
$$

where $0<\delta<\pi / 2$ and $\vartheta_{1}=\min \{\pi / 2+\delta, \pi-\vartheta\}$. Thus, for $\lambda \in \sum_{\vartheta_{1}}$, $\lambda I-\Delta_{\mid D L_{2}(\Omega)}$ is invertible. From Theorem 2.5.2(c) in [11], we have

$$
\begin{aligned}
\| R\left(\lambda: \Delta_{\mid D L_{2}(\Omega)} \|\right. & =\operatorname{Sup}_{\substack{u \in D L_{2}(\Omega) \\
\|u\|_{D L_{2}(\Omega)}=1}}\left\|R\left(\lambda: \Delta_{\mid D L_{2}(\Omega)} \mid\right) u\right\| \\
& \leq \operatorname{Sup}_{\substack{u \in L_{2}(\Omega) \\
\|u\|_{L_{2}(\Omega)}=1}}\|R(\lambda: \Delta) u\|=\|R(\lambda, \Delta)\|_{L_{2}(\Omega)} \leq \frac{M}{|\lambda|} .
\end{aligned}
$$


Now Theorem 2.5.2(c) in [11] implies that $\left\{T(t)_{\mid D L_{2}(\Omega)}: \lambda \geq 0\right\}$ can also be extended to an analytic semigroup on $D L_{2}(\Omega)$. Therefore $\left.\Delta\right|_{D L_{2}(\Omega)}$ is a infinitesimal generator of an analytic semigroup of contraction on $D L_{2}(\Omega)$.

Suppose that $-A$ is the infinitesimal generator of an analytic semigroup $T(t)$ on a Banach space $X$. From the results of Subsection 2.6 in [11], we can define the fraction powers $A^{\alpha}$ for $0 \leq \alpha \leq 1$ and $A^{\alpha}$ is a closed linear invertible operator with domain $D\left(A^{\alpha}\right)$ dense in $X . D\left(A^{\alpha}\right)$ equipped with the norm $\|x\|_{\alpha}=\left\|A^{\alpha} x\right\|$ is a Banach space denoted by $X_{\alpha}$. It is clear that $0<\alpha<\beta$ implies $X_{\alpha} \supset X_{\beta}$ and that the embedding of $X_{\beta}$ into $X_{\alpha}$ is continuous. If $-A=\Delta$ and $\gamma>1 / 2$, then $X_{\gamma} \subset X_{\frac{1}{2}}$ and $D\left((-\Delta)^{\gamma}\right) \subset D\left((-\Delta)^{1 / 2}\right)$, so the norms $\|\bullet\|_{D L_{2}(\Omega)_{\gamma}}$ and $\|\bullet\|_{D L_{2}(\Omega)}$ are equivalent (see p. 291 in [6]), i.e., there exists $L_{0}>0$ such that for any $u \in D\left((-\Delta)^{\gamma}\right)\|\bullet\|_{D L_{2}(\Omega) \frac{1}{2}}$

$$
\left\|(-\Delta)^{\gamma} u\right\|_{D L_{2}(\Omega)}=\|u\|_{D L_{2}(\Omega)_{\gamma}} \leq L_{0}\|u\|_{D L_{2}(\Omega) 1 / 2}
$$

For $u \in D(\Delta)$, we have

$$
\|\nabla u\|_{D L_{2}(\Omega)}=\|-\nabla u\|_{D L_{2}(\Omega)}=\left\|(-\Delta)^{1 / 2} u\right\|_{D L_{2}(\Omega)}=\|u\|_{D L_{2}(\Omega) 1 / 2} \cdot
$$

In [2], Giga and Miyakava proved the following result:

Lemma 5 (Lemma 2.2 in [2]). Let $0 \leq \delta<1 / 2\left|-n\left(1-r^{-1}\right) / 2\right|$. Then

$$
\left\|A^{-\delta} P(u, \nabla) v\right\|_{0, r} \leq M\left\|A^{\vartheta} u\right\|_{0, r}\left\|A^{\omega} v\right\|_{0, r},
$$

with some constant $M=M(\delta, \vartheta, \omega, r)$, provided $\delta+\vartheta+\omega \geq n / 2 r+1 / 2$, $\vartheta>0, \omega>0, \omega+\delta>1 / 2$. 
From the Lemma 5 and the formula (10) we see that if take $n=3, r=2, \delta=0, \vartheta=3 / 4$ and $\omega=3 / 4$, then

$$
\begin{aligned}
\|(u \bullet \nabla) v\|_{D L_{2}(\Omega)} & =\|(u \bullet \nabla) v\|_{L_{2}(\Omega)}=\|(u \bullet \nabla) v\|_{W^{0,2}(\Omega)} \\
& \leq M\left\|(-\Delta)^{\vartheta} u\right\|_{D L_{2}(\Omega)}\left\|(-\Delta)^{\omega} v\right\|_{D L_{2}(\Omega)} \\
& \leq M L_{0}^{2}\|u\|_{D L_{2}(\Omega)_{1 / 2}}\|v\|_{D L_{2}(\Omega)_{1} / 2},
\end{aligned}
$$

with some constant $M$ for any $u, v \in D L_{2}(\Omega)$. Hence we have

Lemma 6. Suppose that $u, v \in D L_{2}(\Omega)$ are velocity fields and $(u \bullet \nabla) v \in D L_{2}(\Omega)$, then

$$
\|(u \bullet \nabla) v\|_{D L_{2}(\Omega)} \leq M L_{0}^{2}\|u\|_{D L_{2}(\Omega)_{1} / 2}\|v\|_{D L_{2}(\Omega)_{1} / 2} .
$$

Assumption (F). Let $X=D L_{2}(\Omega)$ and $U$ be an open subset in $R^{+} \times X_{\alpha}(0<\alpha<1)$. The function $f: U \rightarrow X$ satisfies the Assumption (F) if for every $(t, u) \in U$ there is a neighbourhood $V \subset U$ and constants $L \geq 0,0<\vartheta \leq 1$ such that for all $\left(t_{i}, u_{i}\right) \in V(i=1,2)$

$$
\left\|f\left(t_{1}, u_{1}\right)-f\left(t_{2}, u_{2}\right)\right\| \leq L\left(\left|t_{1}-t_{2}\right|^{\vartheta}+\left\|u_{1}-u_{2}\right\|_{\alpha}\right) .
$$

Lemma 7 (Theorem 6.3.1 in [11]). Let $-A$ be the generator of an analytic semigroup $T(t)$ satisfying $\|T(t)\| \leq M$ and assume that $0 \in \rho(-A)$. If, $0<\alpha<1$ and $f$ satisfies the Assumption ( $\mathrm{F}$ ) then for every initial date $\left(t_{0}, u_{0}\right) \in U$ the initial value problem

$$
\left\{\begin{array}{c}
\frac{d u(t)}{d t}+A u(t)=f(t, u(t)), t \in\left(t_{0}, \infty\right) \\
u\left(t_{0}\right)=u_{0}
\end{array}\right.
$$

has a unique local solution $u \in C\left(\left[t_{0}, t_{1}\right): X\right) \cap C^{1}\left(\left(t_{0}, t_{1}\right): X\right)$, where $t_{1}=t_{1}\left(t_{0}, u_{0}\right)>t_{0}$ 
Now we study the Navier-Stokes initial value problem (1).

A function $u$ which is differentiable almost everywhere on $[0, T]$ such that $u^{\prime} \in L^{1}\left[0, T: D L_{2}(\Omega)\right]$ is called a strong solution of the initial value problem (1) if $u(0)=u_{0}$ and $u$ satisfies (1) a.e. on $[0, T]$.

Let $C^{\mu}\left([0, \infty) ; D L_{2}(\Omega)_{1 / 2}\right)$ denote the space of all Hölder continuous functions on $[0, T]$ with exponent $\mu$ and with values in a Banach space $D L_{2}(\Omega)_{1 / 2}$.

Theorem. The initial value problem (1) has a unique smooth local strong solution if the initial value $u_{0} \in D\left((-\Delta)^{\frac{1}{2}}\right)$ is smooth, divergencefree vector field and $f$ is Hölder continuous about $t$ on $(0, \infty)$ with exponent $\beta_{1}$, i.e., there exist constant $C_{1}$ and $0<\beta_{1}<1$ such that

$$
\|f(s, x)-f(t, x)\| \leq C_{1}|s-t|^{\beta_{1}}, \quad \text { for } \quad s, t \in(0, \infty) \quad \text { and } \quad x \in \Omega .
$$

Proof. We will find that by incorporating the divergence-free condition, we can remove the pressure term from our equation (see p. $271^{3}$ in [3], p. $234_{6}$ and p. $239_{9}$ in [9]). So first we can rewrite (1) into a abstract initial value problem on $D L_{2}(\Omega)$

$$
\left\{\begin{array}{c}
\frac{d u}{d t}=\Delta u+F(t, u(t)), t \in(0, \infty), \\
\left.u\right|_{t=0}=u_{0}, x \in \Omega
\end{array}\right.
$$

where $F(t, u(t))=-(u \bullet \nabla) u+f$ is a abstract function. From Lemma 4, $\Delta$ is the generator of an analytic semigroup $T(t)$ of contraction on $D L_{2}(\Omega)$ and $\|T(t)\| \leq 1$. From Theorem 2.5.2(c) in [11] $0 \in \rho(\Delta)$. 
Let $U$ be the subset of $R^{+} \times D L_{2}(\Omega)_{1 / 2}$ such that $\left(t_{1}, u_{1}\right) \in U$ iff $u_{1}=u\left(t_{1}\right)$ for some $u(t) \in C^{\mu}\left([0, \infty) ; D L_{2}(\Omega)_{1 / 2}\right)$ and $\left(u_{1} \bullet \nabla\right) u_{1} \in D L_{p}(\Omega)$. Let $u_{k}(t, x)=(k, k, k)(t \in[0, \infty), x \in \Omega, k \in R)$. Then $\left(t, u_{k}\right) \in U$ for $t \in[0, \infty)$ and all $k \in R$. For any $u \in D\left((-\Delta)^{\frac{1}{2}}\right)$ let $u(t) \equiv u(t \in[0, \infty))$. Then $u(t) \in C^{\mu}\left([0, \infty) ; D L_{2}(\Omega)_{1 / 2}\right)$. Hence $U$ is not empty.

Let $U_{1}$ be the open kernel of $U$. From Lemma $8 F(t, u(t))=-(u \bullet \nabla)$ $u+f$ is a function: $U_{1} \rightarrow D L_{2}(\Omega)$. For any $\left(t_{1}, u_{1}\right),\left(t_{2}, u_{2}\right) \in U_{1}$, we have $\left(u_{1} \bullet \nabla\right) u_{1},\left(u_{2} \bullet \nabla\right) u_{2} \in D L_{2}(\Omega)$ and

$$
\begin{aligned}
\|\left(u_{1} \bullet \nabla\right) u_{1}- & \left(u_{2} \bullet \nabla\right) u_{2} \|_{D L_{2}(\Omega)} \\
= & \left\|\left(u_{1} \bullet \nabla\right) u_{1}-\left(u_{1} \bullet \nabla\right) u_{2}\right\|_{D L_{2}(\Omega)} \\
& +\left\|\left(u_{1} \bullet \nabla\right) u_{2}-\left(u_{2} \bullet \nabla\right) u_{2}\right\|_{D L_{2}(\Omega)} \\
= & \left.\left\|\left(u_{1} \bullet \nabla\right)\left(u_{1}-u_{2}\right)\right\|_{D L_{2}(\Omega)}+\|\left[\left(u_{1}-u_{2}\right) \bullet \nabla\right)\right] u_{2} \|_{D L_{2}(\Omega)} \\
\leq & M L_{0}^{2}\left(\left\|u_{1}\right\|_{D L_{2}(\Omega)_{1} / 2} \|\left(u_{1}-u_{2}\right)_{D L_{2}(\Omega)_{1} / 2}\right. \\
& \left.+\left\|u_{1}-u_{2}\right\|_{D L_{2}(\Omega)_{1} / 2}\left\|u_{2}\right\|_{D L_{2}(\Omega)_{1} / 2}\right) \\
= & M L_{0}^{2}\left(\left\|u_{1}\right\|_{D L_{2}(\Omega)_{1} / 2}+\left\|u_{2}\right\|_{D L_{2}(\Omega)_{1} / 2}\right)\left\|u_{1}-u_{2}\right\|_{D L_{2}(\Omega)_{1} / 2}
\end{aligned}
$$

We used Lemma 6 in the above third step. Therefore $(u \bullet \nabla) u$ is local Lipschitz continuous. Since $u(t)$ is Hölder continuous about $t$ on $[0, \infty)$ in $D L_{2}(\Omega)_{1 / 2}$, so there is a constant $C_{2}$ and $0<\beta_{2}>1$ such that

$$
\|u(s, x)-u(t, x)\|_{D L_{2}(\Omega)_{1 / 2}} \leq C_{2}|s-t|^{\beta_{2}} \quad \text { for } \quad s, t \in[0, \infty) .
$$


Hence

$$
\begin{aligned}
\left(\|\left(u\left(t_{1}\right)\right.\right. & \bullet \nabla) u\left(t_{1}\right)-\left(u\left(t_{2}\right) \bullet \nabla\right) u\left(t_{2}\right) \|_{D L_{2}(\Omega)} \\
\leq & \left\|\left(u\left(t_{1}\right) \bullet \nabla\right) u\left(t_{1}\right)-\left(u\left(t_{1}\right) \bullet \nabla\right) u\left(t_{2}\right)\right\|_{D L_{2}(\Omega)} \\
& +\left\|\left(u\left(t_{1}\right) \bullet \nabla\right) u\left(t_{2}\right)-\left(u\left(t_{2}\right) \bullet \nabla\right) u\left(t_{2}\right)\right\|_{D L_{2}(\Omega)} \\
= & \left\|\left(u\left(t_{1}\right) \bullet \nabla\right)\left(u\left(t_{1}\right)-u\left(t_{2}\right)\right)\right\|_{D L_{2}(\Omega)} \\
& +\left\|\left[\left(u\left(t_{1}\right)-u\left(t_{2}\right)\right) \bullet \nabla\right] u\left(t_{2}\right)\right\|_{D L_{2}(\Omega)} \\
\leq & M L_{0}^{2}\left(\left\|u\left(t_{1}\right)\right\|_{D L_{2}(\Omega)_{1} / 2}\left\|\left(u\left(t_{1}\right)-u\left(t_{2}\right)\right)\right\|_{D L_{2}(\Omega)_{1} / 2}\right. \\
& \left.+\left\|u\left(t_{1}\right)-u\left(t_{2}\right)\right\|_{D L_{2}(\Omega)_{1} / 2}\left\|u\left(t_{2}\right)\right\|_{D L_{2}(\Omega)_{1} / 2}\right) \\
= & M L_{0}^{2}\left(\left\|u\left(t_{1}\right)\right\|_{D L_{2}(\Omega)_{1} / 2}+\left\|u\left(t_{2}\right)\right\|_{D L_{2}(\Omega)_{1} / 2}\right)\left\|u\left(t_{1}\right)-u\left(t_{2}\right)\right\|_{D L_{2}(\Omega)_{1}} \\
\leq & M L_{0}^{2} C_{2}\left(\left\|u\left(t_{1}\right)\right\|_{D L_{2}(\Omega)_{1} / 2}+\left\|u\left(t_{2}\right)\right\|_{D L_{2}(\Omega)_{1} / 2}\right) \cdot\left|t_{1}-t_{2}\right|^{\beta_{2}} .
\end{aligned}
$$

We used the Lemma 6 in the above third step, and the formula (16) in fifth step.

$$
\begin{aligned}
& \text { If } u_{0} \in D\left((-\Delta)^{\frac{1}{2}}\right) \text {, then }\left(0, u_{0}\right) \in U_{1} \text {. Set } \\
& \qquad V=B_{\varepsilon}\left(0, u_{0}\right)=\left\{(t, u) \in U:|t-0| \leq \varepsilon,\left\|u-u_{0}\right\|_{D L_{2}(\Omega)_{1 / 2}} \leq \varepsilon\right\} .
\end{aligned}
$$

Then for $(t, u) \in V$,

$$
\begin{aligned}
\|u\|_{D L_{2}(\Omega)_{1} / 2} & =\left\|u-u_{0}+u_{0}\right\|_{D L_{2}(\Omega)_{1} / 2} \\
& \leq\left\|u-u_{0}\right\|_{D L_{2}(\Omega)_{1 / 2}}+\left\|u_{0}\right\|_{D L_{2}(\Omega)_{1 / 2}} \\
& \leq \varepsilon+\left\|u_{0}\right\|_{D L_{2}(\Omega)_{1} / 2} .
\end{aligned}
$$


Let $L=\varepsilon+\left\|u_{0}\right\|_{D L_{2}(\Omega)_{1} / 2}, L_{1}=2 M L_{0}^{2} L, L_{2}=2 M L_{0}^{2} L C_{2}$, and $L_{3}=\operatorname{Max}$ $\left(C_{1}, L_{2}, L_{1}\right), \vartheta=\operatorname{Max}\left(\beta_{1}, \beta_{2}\right)$ then from (13), (15) and (17) it follows that for all $\left(t_{i}, u_{i}\right) \in V$

$$
\begin{aligned}
\| F\left(t_{1},\right. & \left.u_{1}\left(t_{1}\right)\right)-F\left(t_{2}, u_{2}\left(t_{2}\right)\right) \|_{D L_{2}(\Omega)} \\
\leq & \left\|F\left(t_{1}, u_{1}\left(t_{1}\right)\right)-F\left(t_{1}, u_{2}\left(t_{1}\right)\right)\right\|_{D L_{2}(\Omega)} \\
& +\left\|F\left(t_{1}, u_{2}\left(t_{1}\right)\right)-F\left(t_{2}, u_{2}\left(t_{2}\right)\right)\right\|_{D L_{2}(\Omega)} \\
= & \left\|\left(u_{1}\left(t_{1}\right) \bullet \nabla\right) u_{1}\left(t_{1}\right)-\left(u_{2}\left(t_{1}\right) \bullet \nabla\right) u_{2}\left(t_{1}\right)\right\|_{D L_{2}(\Omega)} \\
& +\left\|\left(u_{2}\left(t_{1}\right) \bullet \nabla\right) u_{2}\left(t_{1}\right)-\left(u_{2}\left(t_{2}\right) \bullet \nabla\right) u_{2}\left(t_{2}\right)\right\|_{D L_{2}(\Omega)} \\
& +\left\|f\left(t_{1}, x\right)-f\left(t_{2}, x\right)\right\|_{D L_{2}(\Omega)} \\
\leq & 2 M L_{0}^{2} L\left\|u_{1}-u_{2}\right\|_{D L_{2}(\Omega)_{1} / 2}+2 M L_{0}^{2} L C_{2}\left|t_{1}-t_{2}\right|^{\beta_{2}}+C_{1}\left|t_{1}-t_{2}\right|^{\beta_{1}} \\
\leq & \left.L_{1}\left\|u_{1}-u_{2}\right\|_{D L_{2}(\Omega)}\right)_{2}+L_{2}\left|t_{1}-t_{2}\right|^{\beta_{2}}+C_{1}\left|t_{1}-t_{2}\right|^{\beta_{1}} \\
\leq & L_{3}\left(\left|t_{1}-t_{2}\right|^{\vartheta}+\left\|u_{1}-u_{2}\right\|_{D L_{2}(\Omega)_{1} / 2}\right) .
\end{aligned}
$$

Hence $F(t, u(t))$ satisfies the Assumption (F), then by Lemma 7 for every initial data $\left(0, u_{0}\right) \in U_{1}$ the initial value problem (14) has a unique local solution

$$
u(t) \in C\left(\left[0, t_{1}\right): D L_{2}(\Omega)\right) \cap C^{1}\left(\left(0, t_{1}\right): D L_{2}(\Omega)\right),
$$

where $t_{1}=t_{1}\left(u_{0}\right)$.

We consider Equation (3) in integral form

$$
u(t)=e^{t P \Delta} u_{0}+\int_{0}^{t} e^{(t-s) P \Delta} F u(s) d s
$$


In a similar induction way as Theorem 3.9 in [2] or as Theorem $5.1 \mathrm{in}$ [10] we can prove that the solution $u(t, x) \in\left(C^{\infty}\left(\left(0, t_{1}\right] \times \bar{\Omega}\right)\right)^{3} \quad$ (see Appendix). We can also prove directly that $u(t, x)$ is smooth. In fact, the solution (18) of (14) is also the solution of (19). The Theorem 3.4 in [2] mean that as long as the solution of (19) exists, this solution is smooth. From Theorem 3.4 in [2] we have the solution $u(t, x) \in\left(C^{\infty}\left(\left(0, t_{1}\right] \times \bar{\Omega}\right)\right)^{3}$. Substituting $u(t, x)$ into (1) we get the solution $p(t, x)$. We also have $p(t, x) \in C^{\infty}\left(\left(0, t_{1}\right] \times \bar{\Omega}\right)$. It follows from the formula (2) and $u \in D L_{2}(\Omega)$ that the solution $u(t, x)$ is divergence-free. Changing the value of $u$ on $\partial \Omega$ to zero we get a unique smooth local strong solution $u=u(t, x), p(t, x)$ of the Navier-Stokes initial value problem (1).

The above Theorem extents Theorem 1.2 in [3] and Theorem 2.5 in [2]. In another paper, we will prove that if we take $f(t, x)$ to be identically zero and assume that any initial value $u_{0} \in D\left((-\Delta)^{\frac{1}{2}}\right) \cap C^{\infty}\left([0, \infty) \times R^{3}\right)$ satisfies $\left|\partial_{x}^{\alpha} u_{0}(x)\right| \leq C_{\alpha k}(1+|x|)^{-k}$ on $R^{3}$ for any $\alpha$ and $k$, then the Navier-Stokes initial value problem has a global smooth solution with bounded energy.

\section{References}

[1] Andras Batkai, Marjeta Kramar Fijavz and Abdelaziz Rhandi, Positive Operator Semigroups, Birkhauser, 2017.

[2] Y. Giga and T. Miyakava, Solutions in $L_{r}$ of the Navier-Stokes initial value problem, Archive for Rational Mechanics and Analysis 89(3) (1985), 267-281.

DOI: https://doi.org/10.1007/BF00276875

[3] H. Fujita and T. Kato, On the Navier-Stokes initial value problem: I, Archive for Rational Mechanics and Analysis 16(4) (1964), 269-315.

DOI: https://doi.org/10.1007/BF00276188 
[4] T. Kato, Strong $L^{p}$-solution of the Navier-Stokes equation in $R^{m}$, with application to weak solutions, Mathematische Zeitschrift 187(4) (1984), 471-480.

DOI: https://doi.org/10.1007/BF01174182

[5] Klaus-Jochen Engel and Rainer Nagel, One-Parameter Semigroups for Linear Evolution Equations, Springer, 2000.

[6] E. Kreyszig, Introduction Functional Analysis with Applications, John Wiley \& Sons, 1978.

[7] Peter Meyer-Nieberg, Banach Lattices, Springer-Verlag, 1991.

[8] M. Otelbaev, Existence of a strong solution of the Navier-Stokes equation, Mathematical Journal (ISSN 1682-0525) 13(4) (2013), 5-104.

[9] James C. Robinson, Infinite-Dimensional Dynamical Systems, Cambridge University Press, 2001.

[10] Veli B. Shakhmurov, Nonlocal Navier-Stokes problems in abstract function spaces and applications, Nonlinear Analysis: Real World Applications 26 (2015), 19-43.

DOI: https://doi.org/10.1016/j.nonrwa.2015.03.011

[11] A. Pazy, Semigroups of Linear Operators and Applications to Partial Differential Equations, Springer Verlag (1983, reprint in China in 2006).

[12] K. Yosida, Functional Analysis, 6th Edition, Springer Verlag, 1980. 


\section{Appendix}

Lemma 1 (Lemma 3.1 in [2]). Let $u \in D(-\Delta)$ and $-\Delta u \in\left(W^{m, r}(\Omega)\right)^{n}$ $(1<r<\infty)$ for some integer $m \geq 0$, then $u \in\left(W^{m+2, r}(\Omega)\right)^{n}$ and satisfies

$$
\|u\|_{m+2, r} \leq C_{m r}\|-\Delta u\|_{m, r}
$$

with a constant $C_{m r}>0$ independent of $u$ and $-\Delta u$.

Let $C^{\mu}([0, T] ; X)$ denote the space of Hölder continuous functions on $[0, T]$ with exponent $\mu$ and with values in a Banach space $X$. Similarly, let $C^{\mu}((0, T] ; X)$ denote the space of functions which are Hölder continuous on every subinterval $[\varepsilon, T]$ of $(0, T]$, with exponent $\mu$.

Lemma 2 (Lemma 3.2 in [2]). Let $f(t) \in C^{\mu}\left([0, T] ; D L_{2}(\Omega)\right)$, for some $0<\mu<1$. Then the function

$$
v(t)=\int_{0}^{t} e^{-(t-s)(-\Delta)} f(s) d s \in C^{\mu}((0, T] ; D(-\Delta)) \cap C^{1+v}\left((0, T] ; D L_{2}(\Omega)\right),
$$

for every $v$ such that $0<v<\mu$.

Let $P_{r}$ be the continuous projection from $L_{2}(\Omega)$ to $D L_{2}(\Omega)$.

Lemma 3 (Lemma 3.3 in [2]). (i) For $m>3 / r$, there exists a constant $C_{m, r}>0$ such that

$$
\left\|P_{r}(u \bullet \nabla) v\right\|_{m, r} \leq C_{m, r}\|u\|_{m, r}\|v\|_{m+1, r},
$$

for every $u \in\left(W^{m, r}(\Omega)\right)^{3}, v \in\left(W^{m+1, r}(\Omega)\right)^{3}(1<r<\infty)$.

(ii) When $r>3$, we have

$$
\left\|P_{r}(u \bullet \nabla) v\right\|_{0, r} \leq C_{r}\|u\|_{1, r}\|v\|_{1, r},
$$

for $u, v \in\left(W^{1, r}(\Omega)\right)^{3}$. 
We will say that $u(t)$ has property $\left(P_{m}\right)(m \geq 1)$ if

$$
\begin{aligned}
& u^{(m)} \in C^{\mu}\left((0, T] ; D\left((-\Delta)^{\frac{1}{2}}\right),\right. \\
& u^{(j)} \in C^{\mu}\left((0, T] ;\left(W^{m+1-j, 2}(\Omega)\right)^{n}\right), 1 \leq j \leq m-1, \\
& u \in C^{\mu}\left((0, T] ;\left(W^{m+2,2}(\Omega)\right)^{n}\right),
\end{aligned}
$$

for all $\mu, 0<\mu<1 / 2$. Here $u^{(j)}=d^{j} u / d t^{j}$.

Let the solution in the above Theorem be

$$
u(t, x)=T(t) u_{0}+\int_{0}^{t} T(t-s) F\left(s,(-\Delta)^{-\frac{1}{2}} y_{0}(s) d s\right.
$$

Lemma 2 and Lemma 3(i) now imply.

Lemma 4 (Lemma 3.6 in [2]). $u \in C^{\mu}((0, T] ; D(-\Delta))$ and $u^{\prime}=d u / d t$ $\in C^{\mu}\left((0, T] ; D L_{2}(\Omega)\right)$ for all $\mu, 0<\mu<1 / 2$. Moreover $F\left(s,(-\Delta)^{-\frac{1}{2}} y_{0}(s)\right)$ $\in C^{\mu}\left((0, T] ;\left(W^{1,2}(\Omega)\right)^{3}\right)$.

Lemma 5 (Lemma 3.7 in [2]). We have $u^{\prime}=\partial u / \partial x \in C^{\mu}$ $\left((0, T] ; D\left((-\Delta)^{\frac{1}{2}}\right)\right)$ for all $\mu, 0<\mu<1 / 2$.

The proof is similar to Lemma 3.7 in [2].

Since $D\left((-\Delta)^{\frac{1}{2}}\right) \subset\left(W^{1, r}(\Omega)\right)^{3}$, Lemma 1 , Lemma 4 and the identity $u=(-\Delta)^{-1}\left(F u-u^{\prime}\right)$ show that

$$
u \in C^{\mu}\left(\left((0, T] ;\left(W^{3,2}(\Omega)\right)^{3}\right)\right.
$$

Lemma 5 and the above formula (20) show that $u(t)$ has property $(P)_{1}$. 
Lemma 6 (Lemma 3.8 in [2]). $(P)_{m}$ implies $(P)_{m+1}$.

The proof is the same to Lemma 3.8 in [2].

Therefore we can prove the following theorem in a similar way as Theorem 3.9 in [2] or as Theorem 5.1 in [10].

Theorem. The solution in the above Theorem is smooth. 